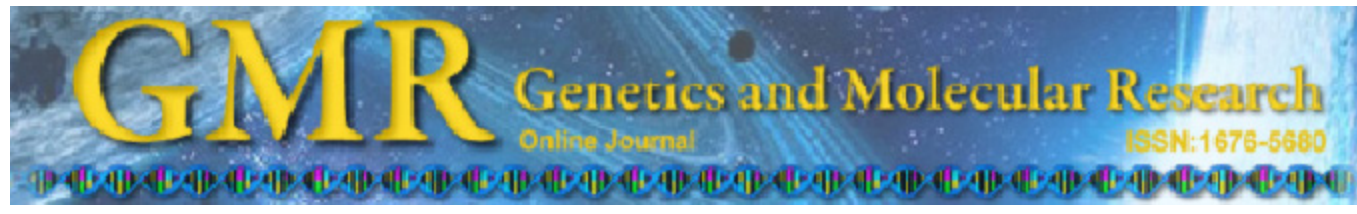

Link to retraction noticed

\title{
Effects of destrin pathway mutations on the gene expression profile
}

\author{
J.N. Xu*, X. Liu*, H. Wang, C.M. Hu, Q.H. Luo and Q.Q. Zhou \\ Department of Vision, Southwest Hospital, Third Military Medical University,
} Chongqing, China

*These authors contributed equally to this study.

Corresponding author: H. Wan

E-mail: wanghuiwhdr@hotmail.com

Genet. Mol. Res. 13 (2): 2628-2637 (2014)

Received January 7, 2013

Accepted June 6, 2013

Published April 8, 201

DOI http://dx.doi.org/10.4238/2014.April.8.5

ABSTRACT. This study aimed to explore the interaction and crosstalk between pathways in response to destrin mutations. All the pathways from the MINT database were downloaded, a protein-protein interaction network was then constructed, and the crosstalk between pathways was investigated, in particular, the overlap of 2 significant pathway analysis results. As expected, the results showed that regulation of the actin cytoskeleton was the significant pathway of destrin mutations in mice. Further analysis indicated that 28 significant pathways crosstalked with the pathway regulating the actin cytoskeleton. Importantly, 3 pathways, including regulation of actin cytoskeleton pathway, pathways in cancer, and the B cell receptor signaling pathway were linked by inositol phosphate metabolism based on crosstalk analysis of Gene Ontology relationships among pathways. All of these pathways have been demonstrated to participate in cytoskeleton dynamics. These findings might provide valuable insights into cytoskeleton dynamic abnormalities in destrin mutations of corneal diseases.

Key words: ADF/Cofilin; Cytoskeleton dynamics; Pathway crosstalk 


\section{INTRODUCTION}

Corneal disease is responsible for $6 \%$ of legal blindness in the United States and is frequently the main cause of bilateral blindness in the world, second only to cataract (Ikeda et al., 2003; Li et al., 2009). Visual loss in many corneal diseases is due to changes in the morphology and function of the corneal epithelial surface, including cell hyperproliferation, inflammation, and angiogenesis (Dawson et al., 2009).

Destrin (also known as actin-depolymerization factor, ADF) is an essential actin regulatory protein of the $\mathrm{ADF} /$ cofilin family that binds to the actin subunits of filamentous actin (F-actin), enhancing the subunit off-rate and promoting filament severing. Thus, this family of proteins is responsible for increasing the turnover of actin filaments and is involved in the regulation of cytoskeleton dynamics (Tokuraku et al., 2001; Maciver and Hussey, 2002). $D{ }^{n}{ }^{\text {cornl }}$ mice represent a spontaneous mutant line that exhibits ocular surface abnormalities shortly after birth and therefore often serves as a good model to study corneal disease. The histology of Dstn ${ }^{\text {cornl }}$ corneas showed that the hyperplastic cornealepithelium expressed an increased level of keratin 14 and involucrin, while the level of keratin 12 was not altered (Zhang et al., 2008). The normal cornea is deficient in blood and lymphatic vessels to maintain corneal transparency, unless severe inflammatory or other strains cause a disruption of the antiangiogenic privilege of the cornea. It has been shown that hemangiogenesis and lymphangiogenesis in the Dstn ${ }^{\text {cornl }}$ cornea depend on vascular endothelial growth factor receptor 3 (VEGFR3) signaling (Cursiefen et al., 2005). Recent reports have suggested that vascularization of Dstn ${ }^{\text {cornl }}$ corneas arises from the lack of soluble VEGFR, sflt-1, which has been proposed as an essential factor for maintenance of ayascularity in a normal cornea. Suppression of endogenous sflt-1 by neutralizing antibodies, RNA interference, or Crelox-mediated gene disruption abolishes corneal avascularity in mice, but recombinant sflt-1 administration restores corneal avascularity in corn1 and Pax6+/- mice (Ambati et al., 2006).

Genome-wide screening of differentially expressed genes (DEGs) in the cornea of $D_{s t n}{ }^{\text {cornl }}$ mice reveals that the expression of a large portion of genes associated with cytoskeletal dynamics was up-regulated (Verdoni et al., 2008). Nearly half of these genes are targets of the serum response factor (SRF), an essential regulator of the actin cytoskeleton (Miano et al., 2007; Miano, 2008). The conditional ablation of Srf in the corneal epithelium of a diseased Dstn ${ }^{\text {cornl }}$ cornea results in the rescue of epithelial cell hyper-proliferation, inflammation, and neovascularization phenotypes (Verdoni et al., 2010). These results indicate that there is an underlying interaction among these DEGs. Given the complex nature of biological systems, pathways often need to function in a coordinated fashion to produce appropriate physiological responses to both internal and external stimuli (Li et al., 2008). Therefore, we performed research on the protein-protein interaction (PPI), significant pathway, and crosstalk between pathways based on the previous study in our institution, with the hope to lay an important theoretical foundation for understanding the molecular mechanism of destrin in corneal diseases.

\section{MATERIAL AND METHODS}

\section{Data sources}

We download all the pathways from KEGG (Kyoto Encyclopedia of Genes and Genomes) (Kanehisa, 2002) and all the PPI datasets from MINT (the Molecular Interaction da- 
tabase) (Ceol et al., 2010), which contains the mouse PPI datasets from IntAct (Aranda et al., 2010), BIOGRID (the Biological General Repository for Interaction Datasets) (Stark et al., 2011), and HPRD (Human Protein Reference Database) (Keshava Prasad et al., 2009).

Next, an ensemble PPI network was constructed by integrating 2 of the above-described PPI databases in mice. A total of 65,851 unique PPI pairs were collected, involving 10,951 unique proteins.

We extracted the gene expression profile data for the Dstn mutations with normal wildtype from Verdoni et al. (2008), which were deposited in NCBI (National Center for Biotechnology Information) GEO (Gene Expression Omnibus database, http://www.ncbi.nlm.nih.gov/ geo/) database (ID: GSE9743). All mouse procedures were performed in accordance with the protocols approved by the Animal Care and Use Committee at the University of WisconsinMadison and conformed to the ARVO statement for the use of animals in Ophthalmic and Vision Research and APS's Guiding Principals in the Care and Use of Animals. The Dstn mutations and wild-type samples with 6 replications in each group were compared.

The Limma package in R language (Smyth, 2004) was used to identify DEGs. Background intensities were adjusted, and the original expression datasets from all conditions were processed into expression estimates using the RMA method with the default settings implemented in R (version 2.12.1) to construct the linear model. Only the DEGs with fold-change values greater than 1.5 and $P$ values less than 0.05 were selected.

\section{Pathway crosstalk analysis}

The crosstalk pathways are defined as those pathways that have overlapping genes and edges. The overlapping genes mean that both pathways are included, and the overlapping edges mean that both pathways included the PPI interaction edges.

To determine the co-expressed significance of a gene pair in disease cases, we used the Pearson correlation coefficient test to calculate the P value.

The $\mathrm{P}$ values were mapped to the nodes and edges of the PPI network collected from MINT. The following formula was used to define a function as the combination of the statistical significance of an interaction according to a scoring matrix. The detailed description can be found in Liu et al. (2010).

$$
\begin{aligned}
& S(e)=f(\operatorname{diff}(x), \operatorname{cor}(x, y), \operatorname{diff}(y)) \\
& =-2 \sum_{i=1}^{k} \log e\left(p_{i}\right)
\end{aligned}
$$

Functions $\operatorname{diff}(x)$ and $\operatorname{diff}(y)$ are differential expression assessments of gene $\mathrm{x}$ and gene y, respectively. Function $\operatorname{cor}(x, y)$ represents the correlation between gene $\mathrm{x}$ and gene $\mathrm{y}$. Method $f$ is a general data integration method that can handle multiple data sources differing in statistical power. When $\mathrm{k}=3, \mathrm{p} 1$ and $\mathrm{p} 2$ are the $\mathrm{P}$ values of differential expression of 2 nodes, and $\mathrm{p} 3$ is the $\mathrm{P}$ value of their co-expression.

\section{Significant pathways analysis}

$$
S p=\sum_{e \in P} S(e)
$$


The scores that are larger than $\mathrm{Sp}$ are used as the significance $\mathrm{P}$ value of pathway $\mathrm{P}$ to describe its importance.

We also used DAVID (Huang et al., 2009) for pathway-enrichment analysis for P values $<0.05$ as input into the DEG dataset.

\section{Crosstalk analysis of relationships among pathways}

The detailed crosstalk analysis of relationships among pathways was then investigated, especially that of overlap between 2 significant pathway analysis results.

To define the interaction significance between pathways, we summarize all the scores of edges S(e) of all non-empty overlaps. Specifically, the interaction score between 2 pathways was estimated by their overlapping status of weighted pathways using the following formula:

$$
C(p i, p j)=\sum_{e \in \mathrm{Oij}} S(e),
$$

where $P i$ and $P j$ are 2 pathways and $O i j$ is their overlap.

To estimate the significance of the overlap between different pathways, we randomly sampled $10^{5}$ times the same size of the 2 pathways from the edges of the pathway network and calculated their overlap scores. The frequency larger than $\mathrm{C}$ is regarded as the interaction significance $\mathrm{P}$ values. Finally, the crosstalk with $\mathrm{P}$ yalues $<0.001$ were considered to be significant.

\section{Significant Gene Ontology (GO)-enrichment analysis of each pathway}

The functional enrichment among proteins in 1 pathway is defined as follows:

where $n$ is the number of nodes in the network, $f$ is the number of proteins annotated with a particular GO function, $m$ is the number of proteins involved in the pathway, and $k$ is the frequency of the GO term. We identified the GO function enrichment of the pathways.

\section{RESULTS}

In this study, the GSE9743 dataset was first downloaded from the GEO database (http://www.ncbi.nlm.nih.gov/geo/), and the R language was then used to calculate the DEGs. Based on the expression profiles, we utilized the PPI dataset and the KEGG pathways to elucidate significant pathways and the crosstalk among these significant pathways.

\section{Significant pathway analysis}

We used Sp to evaluate the importance of pathways (for details, see Material and Methods section). Thirty-six pathways (Table 1 ) were detected with $\mathrm{P}$ values $<0.01$. 


\begin{tabular}{|c|c|c|c|c|c|c|}
\hline ID & Node & Edge & Size & Score & $P$ value & Description \\
\hline mmu04623 & 3 & 49 & 64 & 869.37 & 0 & Cytosolic DNA-sensing pathway \\
\hline mmu05020 & 7 & 55 & 35 & 795.18 & 0 & Prion diseases \\
\hline mmu00052 & 10 & 115 & 29 & 1706.70 & 0 & Galactose metabolism \\
\hline $\mathrm{mmu} 03450$ & 11 & 150 & 13 & 1692.31 & 0 & Non-homologous end-joining \\
\hline mmu04114 & 11 & 31 & 114 & 739.21 & 0 & Oocyte meiosis \\
\hline mmu00100 & 12 & 60 & 18 & 1101.54 & 0 & Steroid biosynthesis \\
\hline mmu00500 & 15 & 131 & 48 & 1630.65 & 0 & Starch and sucrose metabolism \\
\hline $\mathrm{mmu} 00630$ & 19 & 187 & 19 & 2076.68 & 0 & Glyoxylate and dicarboxylate metabolism \\
\hline mmu04621 & 19 & 74 & 58 & 1190.49 & 0 & NOD-like receptor signaling pathway \\
\hline mmu04330 & 27 & 208 & 51 & 2758.92 & 0 & Notch signaling pathway \\
\hline mmu00970 & 34 & 473 & 65 & 6169.44 & 0 & Aminoacyl-tRNA biosynthesis \\
\hline mmu05220 & 34 & 273 & 74 & 3175.71 & 0 & Chronic myeloid leukemia \\
\hline mmu04622 & 47 & 1196 & 69 & 12312.19 & 0 & RIG-I-like receptor signaling pathway \\
\hline mmu03008 & 48 & 825 & 91 & 9438.96 & 0 & Ribosome biogenesis in eukaryotes \\
\hline mmu04662 & 55 & 831 & 77 & 8634.24 & 0 & B cell receptor signaling pathway \\
\hline mmu04810 & 65 & 318 & 218 & 3823.94 & 0 & Regulation of actin cytoskeleton \\
\hline mmu04722 & 79 & 1170 & 132 & 13045.08 & 0 & Neurotrophin signaling pathway \\
\hline mmu04110 & 97 & 883 & 128 & 11079.05 & 0 & Cell cycle \\
\hline mmu04010 & 128 & 521 & 269 & 5473.65 & 0 & MAPK signaling $\mathrm{p}$ \\
\hline mmu04510 & 151 & 1901 & 200 & 19541.14 & 0 & Focal adhesion \\
\hline mmu01100 & 360 & 1207 & 1202 & 12580.05 & & Metabolic pathwa \\
\hline mmu00900 & 8 & 73 & 15 & 899.86 & & Terpenoid backbone biosynthesis \\
\hline mmu04145 & 77 & 712 & 179 & 7202.11 & 1.00 & Phagoson \\
\hline mmu04380 & 79 & 718 & 118 & 7221.60 & $6.00 \mathrm{E}-0$ & Osteoclast differentiation \\
\hline $\mathrm{mmu} 03430$ & 21 & 336 & 22 & 3504.59 & $9.00 \mathrm{E}-05$ & Mismatch repair \\
\hline mmu05200 & 107 & 422 & 326 & 4274.35 & 0.00049 & Pathways in cancer \\
\hline mmu04612 & 13 & 72 & 81 & 828.29 & $5.00 \mathrm{E}-04$ & Antigen processing and presentation \\
\hline mmu05133 & 5 & 9 & 74 & 138.45 & 0.00057 & Pertussis \\
\hline mmu04070 & 2 & 11 & & & 0.00085 & Phosphatidylinositol signaling system \\
\hline mmu04666 & 7 & 23 & & 297.5 & 0.00098 & Fc gamma R-mediated phagocytosis \\
\hline mmu05152 & 12 & 53 & & & 0.00113 & Tuberculosis \\
\hline mmu00562 & 34 & 181 & & & 0.00136 & Inositol phosphate metabolism \\
\hline mmu04672 & 14 & 32 & & & 0.00154 & Intestinal immune network for IgA production \\
\hline mmu04130 & 26 & 154 & & 1610.03 & 0.00359 & SNARE interactions in vesicular transport \\
\hline mmu04115 & 15 & & & & 0.00851 & p53 signaling pathway \\
\hline mmu04916 & 7 & 3 & & 164.38 & 0.00877 & Melanogenesis \\
\hline
\end{tabular}

The Limma package was used to detect 120 DEGs (for details, see Material and Methods section). Using DAVID with the DEGs, several pathways were identified. However, we only found 4 significant pathways: regulation of actin cytoskeleton (mmu04810) with a $\mathrm{P}$ value $=0.01$, leukocyte transendothelial migration $(\mathrm{mmu} 04670)$ with a $P$ value $=0.001$, tight junction (mmu04530) with a $P$ value $=0.017$, and arrhythmogenic right ventricular cardiomyopathy (mmu05412) with a P value $=0.04$.

Remarkably, only 1 overlap of a significant pathway (regulation of actin cytoskeleton, mmu04810, marked red in Table 1), was detected in the Dstn mutations.

\section{Crosstalk among the pathways}

Further, we exploited the pathway crosstalk between regulation of actin cytoskeleton (mmu04810) and other significant pathways using the overlapping score. We found that 28 significant pathways cross-talked to the mmu04810 pathway (Table 2). 
Table 2. Crosstalk between mmu04810 and corneal related pathways.

\begin{tabular}{|c|c|c|c|}
\hline PathID_A & PathID_B & PathID_B_name & $P$ value \\
\hline mmu04810 & mmu00052 & Galactose metabolism & 0 \\
\hline mmu04810 & mmu00100 & Steroid biosynthesis & 0 \\
\hline $\mathrm{mmu} 04810$ & $\mathrm{mmu} 00500$ & Starch and sucrose metabolism & $6.00 \mathrm{E}-05$ \\
\hline mmu04810 & mmu00970 & Aminoacyl-tRNA biosynthesis & 0 \\
\hline mmu04810 & mmu01100 & Metabolic pathways & 0 \\
\hline mmu04810 & mmu03008 & Ribosome biogenesis in eukaryotes & 0 \\
\hline mmu04810 & $\mathrm{mmu} 03450$ & Non-homologous end-joining & 0.0018 \\
\hline mmu04810 & mmu04070 & Phosphatidylinositol signaling system & 0 \\
\hline mmu04810 & $\mathrm{mmu} 04110$ & Cell cycle & 0 \\
\hline mmu04810 & mmu04114 & Oocyte meiosis & 0 \\
\hline mmu04810 & mmu04115 & p53 signaling pathway & 0 \\
\hline mmu04810 & mmu04145 & Phagosome & 0 \\
\hline mmu04810 & mmu04330 & Notch signaling pathway & 0 \\
\hline $\mathrm{mmu} 04810$ & $\mathrm{mmu} 04380$ & Osteoclast differentiation & 0.00026 \\
\hline mmu04810 & $\mathrm{mmu} 04510$ & Focal adhesion & 0.00032 \\
\hline $\mathrm{mmu} 04810$ & $\mathrm{mmu} 04612$ & Antigen processing and presentation & $1.00 \mathrm{E}-05$ \\
\hline mmu04810 & mmu04621 & NOD-like receptor signaling pathway & 0 \\
\hline mmu04810 & mmu04622 & RIG-I-like receptor signaling pathway & 0 \\
\hline mmu04810 & $\mathrm{mmu} 04623$ & Cytosolic DNA-sensing pathway & 0 \\
\hline mmu04810 & mmu04666 & Fc gamma R-mediated phagoeytosis & 0.00258 \\
\hline mmu04810 & mmu04672 & Intestinal immune network $\mathrm{f}$ & 0.00426 \\
\hline mmu04810 & $\mathrm{mmu} 04722$ & Neurotrophin signaling pathway & $2.00 \mathrm{E}-05$ \\
\hline mmu04810 & mmu04742 & Taste transduction & 0.00654 \\
\hline mmu04810 & mmu04916 & Melanogenesis & 0 \\
\hline mmu04810 & mmu05020 & Prion diseases & $2.00 \mathrm{E}-05$ \\
\hline mmu04810 & mmu05140 & Leishmaniasis & 0 \\
\hline mmu04810 & mmu05152 & Tuberculosis & 0.00738 \\
\hline mmu04810 & mmu05220 & Chronic myeloid leuke & 0 \\
\hline
\end{tabular}

Only high correlated pathways to the mmu04810 were listed in Table 2. The first two columns are the correlated pathways. The third column is the pathway name of the second column. The last column is the P value of two correlated pathways.

\section{Crosstalk of GO relationships among pathways}

For detailed analysis of the crosstalk between the significant pathways, we used the hypergeometric test to identify the significant GO terms of each pathway, with $\mathrm{P}$ values $<0.05$. The results of the top $5 \mathrm{GO}$ terms in parts of the pathways were used to construct the connection among the pathways. In Figure 1, regulation of actin cytoskeleton (mmu04810) connected with inositol phosphate metabolism (mmu00562) through cell adhesion (GO: 007155), with a crosstalk $P$ value $<0.01$.

Inositol phosphate metabolism (mmu00562), pathways in cancer (mmu05200), and B cell receptor signaling pathway (mmu04662) were connected through signal transduction (GO: 007165).

We also found that significant pathways, such as chronic myeloid leukemia (mmu05220), tuberculosis (mmu05152), MAPK signaling pathway (mmu04010), focal adhesion (mmu04510), and cytosolic DNA-sensing pathway (mu04623), were connected with mammary gland epithelial cell proliferation (GO: 0033598), MAPK import into nucleus (GO: 0000189), negative regulation of apoptosis (GO: 0043066), and induction of apoptosis (GO: 006917).

Based on the significant GO enrichments, we predicted the crosstalk between the GO biological processes during the disease development among the pathways. 


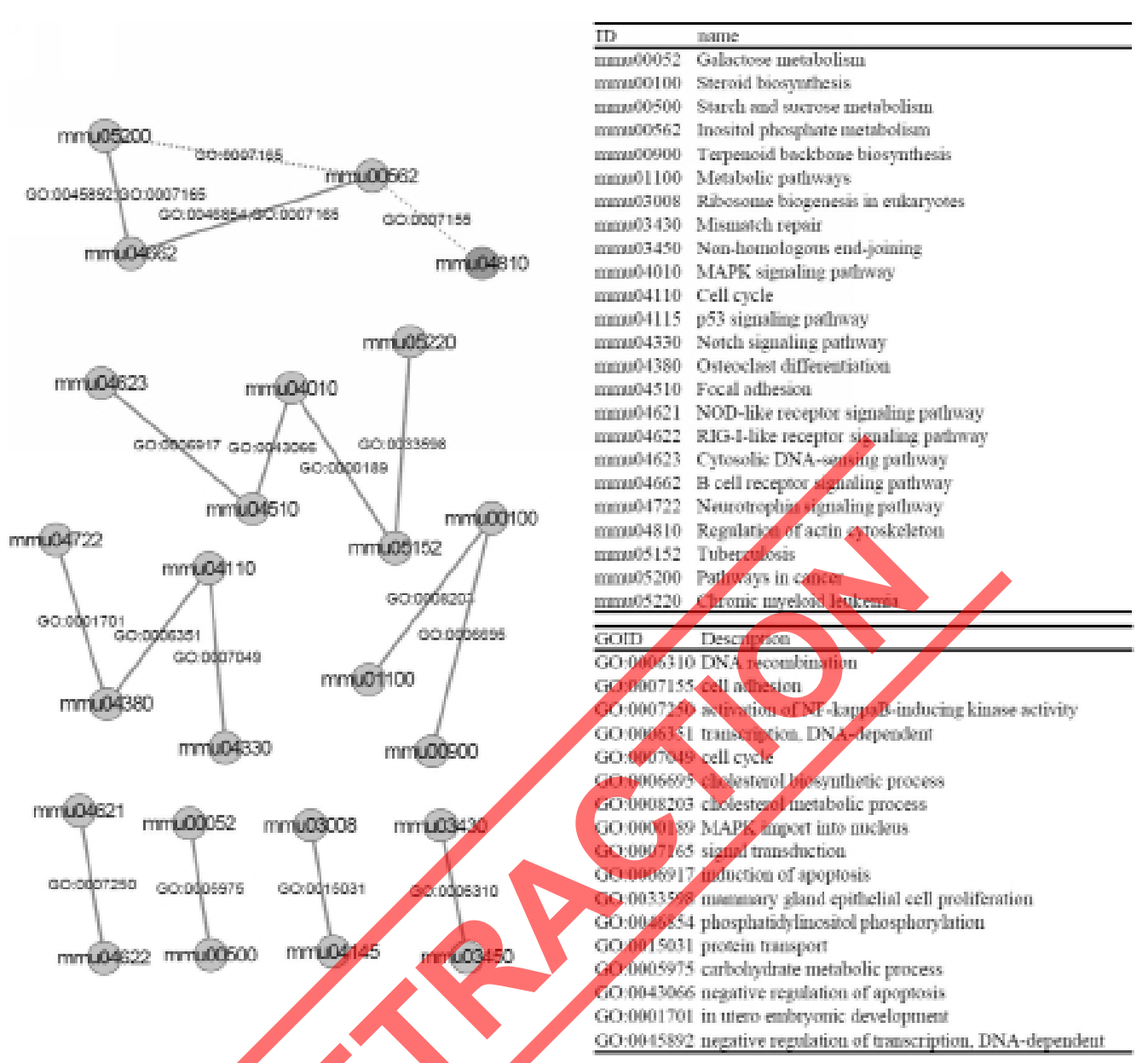

Figure 1. Crosstalk with the overlap of top 5 GO terms of pathways. Significantly enriched GO biological processes are identified in eyery pathway. The edge of each pair of pathways represents the connection with the same GO terms. The solid lines mean the crosstalk's $P$ value $<0.01$ and the dotted lines mean the $P$ value $>0.01$.

\section{DISCUSSION}

Dstn $^{\text {corn } 1}$ mice exhibit an actin dynamic defect in corneal epithelial cells, offering an in vivo model to investigate cellular mechanisms affected by the Dstn mutation and resultant actin dynamic abnormalities. Microarray analysis using the cornea from Dstn ${ }^{\text {cornl }}$ and wild-type mice demonstrated that Dstn mutations have a strong influence on the gene expression profile, especially on the actin cytoskeleton regulator. As anticipated, regulation of the actin cytoskeleton (mmu04810) was also a significant pathway in our analysis. Furthermore, 28 significant pathways cross-talked to the mmu04810 pathway and were identified using the overlapping score (Table 2). Importantly, crosstalk analysis of GO relationships among pathways indicated that the mmu04810 pathway was indirectly connected to the mmu05200 pathway (pathways in cancer) and the mmu04662 pathway (B cell receptor signaling pathway) in a mmu00562 
pathway-mediated manner (inositol phosphate metabolism). The results indicate that there are interaction relationships among these 4 pathways, which is in accordance with previous reports as follows.

Recent studies demonstrated that the organization and dynamics of the actin cytoskeleton could be regulated by the phosphoinositide pathway at several levels, such as phosphatidylinositol-3,4,5-trisphosphate (PIP3), phosphatidylinositol-4,5-bisphosphate (PIP2), and the enzymes catalyzing the production or hydrolysis of these lipids. Therefore, crosstalk between regulation of actin cytoskeleton and the phosphoinositide pathway is expected. Among the different PIs, $\mathrm{PI}(4,5) \mathrm{P} 2$ is the best-characterized regulator of the actin cytoskeleton. $\mathrm{PI}(4,5) \mathrm{P} 2$ interacts directly with several actin-binding proteins, such as ADF/ cofilin, to regulate the activities of the actin-binding proteins (Zhao et al., 2010). Typically, $\mathrm{PI}(4,5) \mathrm{P} 2$ inhibits actin-binding proteins that promote actin filament disassembly and activates proteins that induce actin filament assembly (van Rheenen et al., 2007). Therefore, $\mathrm{PI}(4,5) \mathrm{P} 2$ is considered a promoter of the formation of actin filament structures beneath the plasma membrane and other phosphoinositide-rich membrane organelles. This is supported by a number of studies demonstrating that an increased plasma membrane PI(4,5)P2 level induces actin filament assembly in mammalian cells, while sequestration of PI $(4,5) \mathrm{P} 2$ leads to a defective cortical actin cytoskeleton (Saarikangas et al,, 2010).

One study has demonstrated that there is a relationship between the regulation of the actin cytoskeleton and $\mathrm{B}$ cell receptor (BCR) signaling in the antigen processing and presentation process. Bruton's tyrosine kinase (Btk) is one linker connecting BCR signaling to actin dynamics. Using xid mice and a Btk inhibitor, BCR engagement increases actin polymerization and Wiskott-Aldrich syndrome protein (another actin-binding protein, similar to ADF/ cofilin) activation in a Btk-dependent manner. Concurrently, Btk-dependent increases based on the level of PIP2, and phosphorylated Vay is observed upon BCR engagement. Thus, the BCR-triggered signaling regulates the dynamics of the actin cytoskeleton through WASP in a Btk-dependent manner (Sharma et al., 2009).

Cell hyperproliferation, inflammation, and angiogenesis are biological processes central to the pathogenesis of corneal disease, as well as other conditions including tumorigenesis and chronic inflammatory disorders. Therefore, pathways in cancer may be involved in the regulation of the actin cytoskeleton. In the past decade, many signaling pathways have been identified to be associated with cancer development, such as Ras/MAPK, MAPK/ERK, TGF- $\beta$, and PI3K (Dreesen and Brivanlou, 2007). These pathways have all been proposed to influence the regulation of the actin cytoskeleton. For example, the Ras/MAPK pathway is likely the critical pathway involved in cytoskeleton disruption during Ras transformation. Oncogenic Ras can specifically target the actin-based cytoskeleton and achieve morphological transformation of the cells by down-regulation of structural components of the cytoskeleton and inhibition of ROCKI/Rho kinase-dependent pathways (Pawlak and Helfman, 2002; Samaj et al., 2004). TGF- $\beta$, via Smad and p38Mapk, up-regulates expression of actin-binding proteins such as ADF/cofilin to regulate the actin cytoskeleton and cell motility in epithelial cells (Bakin et al., 2004; Vardouli et al., 2005; Moustakas and Heldin, 2008). One study demonstrated that the JNK and PI3K signaling cascades initiate in the early stages of angiogenesis through the reorganization of the actin cytoskeleton to increase production and activation of MMP-2. However, JNK mainly regulates the mRNA expression of MMP-2 and MT1-MMP, whereas PI3K regulates protein levels (Ispanovic and Haas, 2006). 
In conclusion, in this paper, a network-based approach was used to analyze the crosstalk between corneal related pathways. The crosstalk between the pathways was identified and analyzed using PPI datasets and expression profiles. The results are consistent with prior knowledge of actin dynamics. The crosstalk of pathways presents new alternative insights for corneal disease. As a comprehensive and system-wide analysis, our research may provide evidence for corneal disease and complements traditional component-based approaches.

\section{REFERENCES}

Ambati BK, Nozaki M, Singh N, Takeda A, et al. (2006). Corneal avascularity is due to soluble VEGF receptor-1. Nature 443: 993-997.

Aranda B, Achuthan P, Alam-Faruque Y, Armean I, et al. (2010). The IntAct molecular interaction database in 2010. Nucleic Acids Res. 38: D525-D531.

Bakin AV, Safina A, Rinehart C, Daroqui C, et al. (2004). A critical role of tropomyosins in TGF-beta regulation of the actin cytoskeleton and cell motility in epithelial cells. Mol. Biol. Cell 15: 4682-4694.

Ceol A, Chatr AA, Licata L, Peluso D, et al. (2010). MINT, the molecular interaction database: 2009 update. Nucleic Acids Res. 38: D532-D539.

Cursiefen C, Ikeda S, Nishina PM, Smith RS, et al. (2005). Spontaneous corneal hem-and lymphangiogenesis in mice with destrin-mutation depend on VEGFR3 signaling. Am. J. Pathol. 166:1367-1377.

Dawson DG, Geroski DH and Edelhauser HF (2009). Corneal Endothelium: Structure and Function in Health and Disease. In: Corneal Surgery: Theory, Technique and Tissue (Frederick S. Brightbill, eds.). Mosby, St. Louis, 57.

Dreesen O and Brivanlou AH (2007). Signaling pathways in cancer and embryonic stem cells. Stem. Cell Rev. 3: 7-17.

Huang DW, Sherman BT and Lempicki RA (2009). Systematic and integrative analysis of large gene lists using DAVID bioinformatics resources. Nat. Protoc. 4: 44-57.

Ikeda S, Cunningham LA, Boggess D, Hawes N, et al. (2003). Aberrant actin cytoskeleton leads to accelerated proliferation of corneal epithelial cells in mice deficient for destrin (actin depolymerizing factor). Hum. Mol. Genet. 12: 1029-1037.

Ispanovic E and Haas TL (2006). JNK and PI3K differentially regulate MMP-2 and MT1-MMP mRNA and protein in response to actin cytoskeleton reorganization in endothelial cells. Am. J. Physiol. Cell Physiol. 291: C579-C588.

Kanehisa M, Goto S, Kawashima S and Nakaya A (2002). The KEGG databases at GenomeNet. Nucleic Acids Res. 30: 42-46.

Keshava Prasad TS, Goel R, Kandasamy K, Keerthikumar S, et al. (2009). Human Protein Reference Database - 2009 update. Nucleic Acids Res. 37: D767-D772.

Li Y, Agarwal P and Rajagopalan D (2008). A global pathway crosstalk network. Bioinformatics 24: 1442-1447.

Li Z, Cui H, Zhang L, Liu P, et al. (2009). Prevalence of and associated factors for corneal blindness in a rural adult population (The Southern Harbin Eye Study). Curr. Eye Res. 34: 646-651.

Liu ZP, Wang Y, Zhang XS and Chen L, (2010). Identifying dysfunctional crosstalk of pathways in various regions of Alzheimer's disease brains. BMC Syst. Biol. 4 (Suppl 2): S11.

Maciver SK and Hussey PJ (2002). The ADF/cofilin family: actin-remodeling proteins. Genome Biol. 3: reviews3007.

Miano JM (2008). SRF'ing the actin cytoskeleton with no destrin. Physiol. Genomics 34: 6-8.

Miano JM, Long X and Fujiwara K (2007). Serum response factor: master regulator of the actin cytoskeleton and contractile apparatus. Am. J. Physiol. Cell Physiol. 292: C70-C81.

Moustakas A and Heldin CH (2008). Dynamic control of TGF-beta signaling and its links to the cytoskeleton. FEBS Lett. 582: 2051-2065.

Pawlak G and Helfman DM (2002). Post-transcriptional down-regulation of ROCKI/Rho-kinase through an MEKdependent pathway leads to cytoskeleton disruption in Ras-transformed fibroblasts. Mol. Biol. Cell 13: 336-347.

Saarikangas J, Zhao H and Lappalainen P (2010). Regulation of the actin cytoskeleton-plasma membrane interplay by phosphoinositides. Physiol. Rev. 90: 259-289.

Samaj J, Baluska F and Hirt H (2004). From signal to cell polarity: mitogen-activated protein kinases as sensors and effectors of cytoskeleton dynamicity. J. Exp. Bot. 55: 189-198.

Sharma S, Orlowski G and Song W (2009). Btk regulates B cell receptor-mediated antigen processing and presentation by controlling actin cytoskeleton dynamics in B cells. J. Immunol. 182: 329-339.

Smyth GK (2004). Linear models and empirical Bayes methods for assessing differential expression in microarray experiments. Stat. Appl. Genet. Mol. Biol. 3: Article3.

Stark C, Breitkreutz BJ, Chatr-Aryamontri A, Boucher L, et al. (2011). The BioGRID Interaction Database: 2011 update. 
Nucleic Acids Res. 39: D698-D704.

Tokuraku K, Okamoto S, Katsuki M, Nakagawa H, et al. (2001). The actin-depolymerizing factor destrin has an actinstabilizing domain. Biochem. Cell Biol. 79: 773-778.

van Rheenen J, Song X, van Roosmalen W, Cammer M, et al. (2007). EGF-induced PIP2 hydrolysis releases and activates cofilin locally in carcinoma cells. J. Cell Biol. 179: 1247-1259.

Vardouli L, Moustakas A and Stournaras C (2005). LIM-kinase 2 and cofilin phosphorylation mediate actin cytoskeleton reorganization induced by transforming growth factor-beta. J. Biol. Chem. 280: 11448-11457.

Verdoni AM, Aoyama N, Ikeda A and Ikeda S (2008). Effect of destrin mutations on the gene expression profile in vivo. Physiol. Genomics 34: 9-21.

Verdoni AM, Schuster KJ, Cole BS, Ikeda A, et al. (2010). A pathogenic relationship between a regulator of the actin cytoskeleton and serum response factor. Genetics 186: 147-157.

Zhang W, Zhao J, Chen L, Urbanowicz MM, et al. (2008). Abnormal epithelial homeostasis in the cornea of mice with a destrin deletion. Mol. Vis. 14: 1929-1939.

Zhao H, Hakala M and Lappalainen P (2010). ADF/cofilin binds phosphoinositides in a multivalent manner to act as a PIP(2)-density sensor. Biophys. J. 98: 2327-2336.

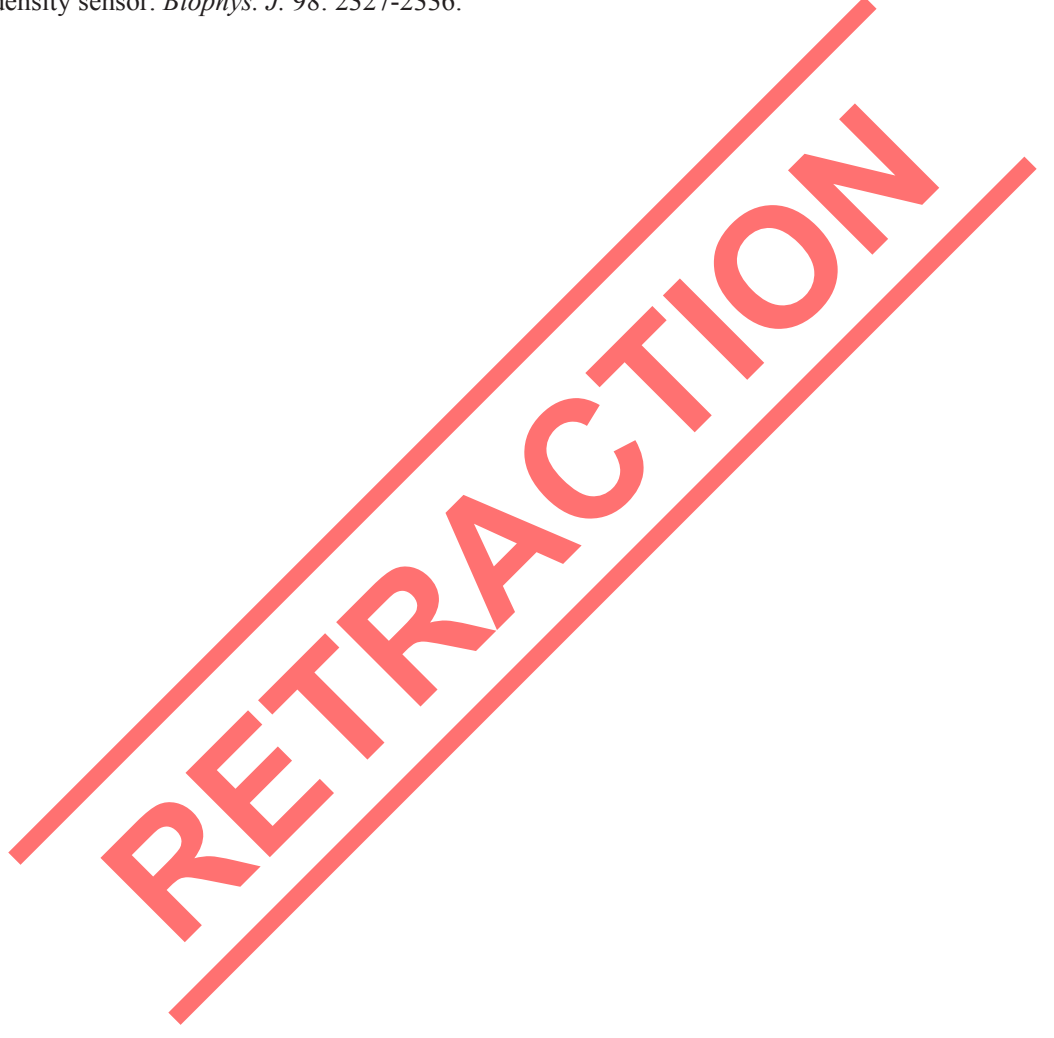

Genetics and Molecular Research 13 (2): 2628-2637 (2014) 